\title{
Enfermeiros e os cuidados paliativos em oncologia: uma revisão integrativa da
}

\section{literatura}

\author{
Nurses and palliative care in oncology: an integrative literature review \\ Enfermeras y cuidados paliativos en oncología: una revisión bibliográfica integradora
}

Recebido: 26/11/2020 | Revisado: 29/11/2020 | Aceito: 12/03/2021 | Publicado: 18/03/2021

Jheniffer Otilia Costa

ORCID: https://orcid.org/0000-0002-4360-1418 Universidade do Vale do Taquari, Brasil

E-mail: jheniffer.costa@universo.univates.br

Fernanda dos Santos

ORCID: https://orcid.org/0000-0001-9153-665X Universidade do Vale do Taquari, Brasil E-mail: fernanda.santos5@univates.br

Paula Michele Lohmann

ORCID: https://orcid.org/0000-0002-8429-9155 Universidade do Vale do Taquari, Brasil E-mail: paulalohmann@univates.br

Caroline Bernardes

ORCID: https://orcid.org/0000-0002-3168-1473 Universidade do Vale do Taquari, Brasil E-mail: bernardes.caroline@gmail.com

\begin{abstract}
Resumo
Trata-se de uma revisão integrativa cujo objetivo é analisar artigos sobre a Preparação do enfermeiro frente a pacientes oncológicos em estado paliativo dos últimos cinco anos. O levantamento foi realizado nos bancos de dados LILACS e SciELO, no mês de agosto de 2019. Após aplicação dos critérios de inclusão e exclusão selecionaram-se cinco artigos para análise na íntegra. Visto como resultados que a maioria das revistas utilizadas foram da enfermagem onde seus enfoques foram: cuidado paliativo na terapia intensiva, cuidado paliativo ao idoso, entendimento dos enfermeiros sobre cuidados paliativos, saber dos acadêmicos de enfermagem com relação aos cuidados paliativos e os sentimentos envolvidos na assistência. Constatou-se que se faz pertinente realizar pesquisas que abordem a preparação do enfermeiro frente a pacientes oncológicos em estado paliativo e a correlação entre técnica e suporte emocional.
\end{abstract}

Palavras-chave: Cuidados paliativos; Cuidados paliativos na terminalidade da vida; Profissionais de enfermagem.

\begin{abstract}
This is an integrative review whose objective is to analyze articles on Nurse Preparation against palliative cancer patients in the last five years. The survey was performed in the LILACS and SciELO databases, in August 2019. After applying the inclusion and exclusion criteria, five articles were selected for full analysis. As a result, most of the journals used were from nursing, where their focuses were: palliative care in intensive care, palliative care for the elderly, nurses' understanding of palliative care, knowledge of nursing students regarding palliative care and the feelings involved in palliative care. assistance. It was found that it is pertinent to conduct research that addresses the preparation of nurses in relation to palliative cancer patients and the correlation between technique and emotional support.
\end{abstract}

Keywords: Palliative care; Hospice care; Nurse practitioners.

\section{Resumen}

Esta es una revisión integradora cuyo objetivo es analizar artículos sobre la preparación de enfermeras contra pacientes con cáncer paliativo en los últimos cinco años. La encuesta se realizó en las bases de datos LILACS y SciELO, en agosto de 2019. Después de aplicar los criterios de inclusión y exclusión, se seleccionaron cinco artículos para un análisis completo. Como resultado, la mayoría de las revistas utilizadas fueron de enfermería, donde se centraron en: cuidados paliativos en cuidados intensivos, cuidados paliativos para ancianos, comprensión por parte de las enfermeras de los cuidados paliativos, conocimiento de los estudiantes de enfermería sobre los cuidados paliativos y los sentimientos relacionados con los cuidados paliativos. Asistencia. Se encontró que es pertinente realizar investigaciones que aborden la preparación de enfermeras en relación con pacientes con cáncer paliativo y la correlación entre la técnica y el apoyo emocional.

Palabras clave: Cuidados paliativos; Cuidados paliativos al final de la vida; Enfermeras practicantes. 


\section{Introdução}

Câncer é o nome dado a um conjunto de mais de 100 doenças que têm em comum o crescimento desordenado e rápido de células, que invadem tecidos e órgãos por meio de metástases. Essa doença afeta todas as faixas etárias, culturas, etnias, sexos e classes sociais. A sua maior suscetibilidade está relacionada, sobretudo, a fatores extrínsecos: o ambiente, em particular a ocupação, dieta, estresse e hábitos de vida (Brasil, 2018).

Por se tratar de uma doença complexa, todas as etapas do tratamento oncológico devem estar contempladas com os princípios do Sistema Único de Saúde (SUS) estabelecidos e garantidos por meio da Lei 8.080 de 1990 - Lei Orgânica da Saúde. Neste sentido, em relação ao câncer, destaca-se o princípio da integralidade, em que o ser humano deve ser visto de maneira integral, ou seja, de forma holística em todos os níveis de saúde. Desta forma, considerar a integralidade do indivíduo, dos serviços e dos cuidados, deve incluir necessariamente um manejo adequado de sintomas de sofrimentos relacionados ao câncer (físicos, psíquicos, espirituais e/ou sociais) e com os processos de morte e morrer, tão importantes e fundamentais quanto outros tratamentos (Floriani \& Schramm, 2008).

Nesta linha de pensamento inserem-se os cuidados ao paciente em estado paliativo, definidos como o conjunto de medidas terapêuticas que visam diminuir as repercussões negativas da doença sobre o bem-estar do indivíduo, seja em ambiente hospitalar ou domiciliar (Monteiro, Oliveira, \& Vall, 2010). Em relação à sua definição, o termo palliare tem sua origem no latim significando proteger, amparar, cobrir e abrigar, tendo como foco central o cuidar e não apenas curar. Logo, a paliação expressa toda medida resultante do alívio do sofrimento do paciente oncológico (Melo \& Figueiredo, 2006).

Em consonância, pesquisa do jornal O Globo, que avaliou os cuidados paliativos disponibilizados aos pacientes terminais em 80 países do mundo, o Brasil ficou em 42 lugar no "Índice de qualidade de morte 2015", um relatório feito pela consultoria britânica Economist Intelligence Unit (EIU). Este estudo analisou a qualidade de hospitais, quantidade de pessoas dedicadas a esse trabalho, suas qualificações e o acesso da população aos cuidados paliativos. Também foi destacado a necessidade de os países estabelecerem parcerias com universidades e organizações sem fins lucrativos, para conseguir alcançar um patamar de qualidade aos cuidados com pacientes terminais (O Globo, 2015).

A equipe que cuida e atua no tratamento paliativo, em que se tem a figura do enfermeiro, como peça chave no cuidado e tratamento destes pacientes, se dá por meio da aplicação de habilidades voltadas para a avaliação sistemática dos sinais e sintomas e no auxílio ao estabelecimento de prioridades do cliente, em que se tem um ambiente genuíno para a prática da enfermagem (Academia Nacional de Cuidados Paliativos, 2009). Tendo em vista tal importância, se faz necessário ao profissional de enfermagem o conhecimento em relação à terminalidade, e toda repercussão que esta fase tem no indivíduo, família e demais envolvidos. Este momento do tratamento ao paciente com câncer pode gerar, dentre tantos sentimentos, angústia e inutilidade, em que se deve buscar a transformação deste processo vital controlando o sofrimento (Bernardes, Bitencourt, Parker, Luz, \& Vargas, 2014).

Logo, a enfermagem que ocupa lugar de destaque nos serviços de saúde, deve estar apta para abarcar o binômio pacientefamília e dispor de conhecimentos e habilidades suficientes para cuidar do ser humano na fase mais importante da vida, o morrer. Acima de tudo deve estar preparado técnica e psicologicamente para proporcionar um cuidado cada vez mais humano e pautado nos princípios do SUS. Dessa maneira, pesquisas que abordam este tema são relevantes em todos os âmbitos da saúde. Logo, a presente pesquisa objetivou realizar uma revisão integrativa na literatura com base na seguinte questão norteadora: Qual a preparação do enfermeiro frente a pacientes oncológicos em estado paliativo?

\section{Metodologia}

O presente estudo trata-se de uma revisão de literatura do tipo integrativa. A busca bibliográfica ocorreu nas bases de dados Literatura Latino-Americana e do Caribe em Ciências da Saúde (LILACS) e Scientific Electronic Library Online (SciELO) 
no período de agosto de 2019. Como estratégia de busca foram utilizadas as palavras-chave devidamente consultadas no Descritores em Ciências da Saúde (DeCS): "Cuidados Paliativos", "Cuidados Paliativos na Terminalidade da Vida" e "Profissionais de Enfermagem".

Foram adotados os seguintes critérios de inclusão: artigos brasileiros, publicados nos últimos cinco anos, aplicado com seres humanos e disponíveis na íntegra. E de exclusão: revisão integrativa, artigos que usaram como amostra apenas pacientes e cuidados paliativos acerca de outras patologias.

Atendendo os critérios, foram identificados artigos sete no LILACS e dois artigos no SciELO. Após a leitura, seis artigos foram eliminados por não corresponderem à temática proposta, totalizando 5 artigos, conforme o fluxograma 1 .

Figura 1 - Fluxograma da seleção de artigos que compõem a revisão integrativa - Lajeado, Rio Grande do Sul, Brasil, 2019.

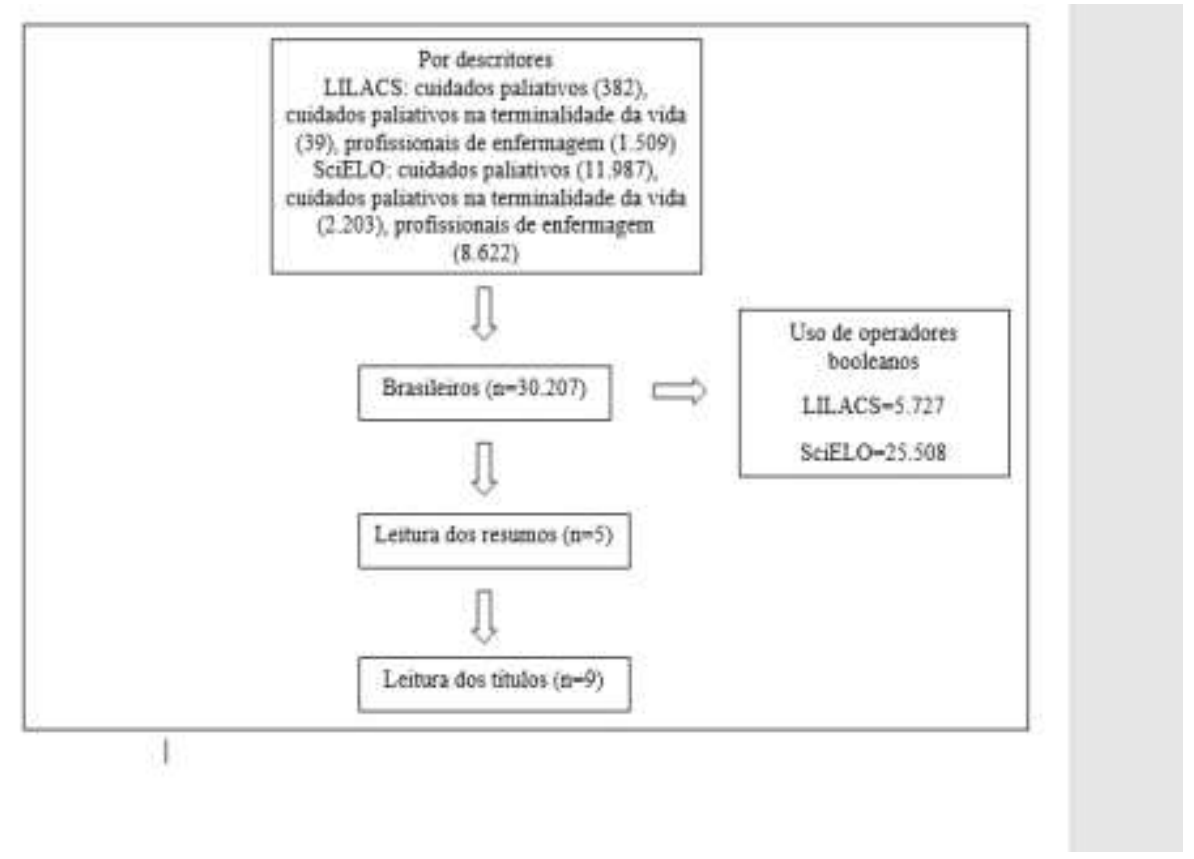

Fonte: Dados da pesquisa (2020).

\section{Resultados e Discussão}

Total de amostras dos artigos foram 122 pessoas, dentre eles: 58 enfermeiros, 8 médicos, 35 técnicos de enfermagem, 16 acadêmicos de enfermagem, 3 fisioterapeutas e 2 nutricionistas. Foram publicados por enfermeiros, doutores em enfermagem, mestres em enfermagem, psicólogo e acadêmicos de enfermagem. Dados que demonstram e sustentam a tese de que a enfermagem é fundamental nos serviços de saúde, bem como o profissional que está diretamente ligado ao cuidado com os pacientes.

Paralelamente, sabe-se que a enfermagem como profissão majoritária nos serviços de saúde assume papel de destaque nos cuidados, porém para gerir um cuidado holístico ao paciente oncológico é importante a reciclagem e preparação deste profissional. A academia nem sempre oferece uma preparação técnica e psicológica para estes profissionais, sendo necessário uma busca contínua de conhecimentos e aprendizagem para poder dar conta destes cuidados.

Os técnicos de enfermagem representam a maioria da equipe de enfermagem, e são os profissionais que atuam diretamente com o paciente durante todo o tratamento, e que consequentemente constroem laços com os mesmos. Porém, nenhum dos artigos foi publicado por técnicos de enfermagem, apenas foram utilizados como amostra da pesquisa em um dos artigos, o que faz questionar sobre a valorização destes profissionais quanto ao cuidado do paciente oncológico em estado paliativo.

Os cinco artigos analisados nesta revisão são oriundos do Brasil, sendo três deles aplicados em hospitais do estado do 
Rio de Janeiro, um no Ceará e um do Piauí. Concernente à fonte, os artigos foram publicados em três periódicos de enfermagem: Acta Paulista de Enfermagem, Texto \& Contexto Enfermagem e Revista online de pesquisa: Cuidado é fundamental. Com relação ao ano de publicação, identificou-se que três deles foram publicados no ano de 2017 e dois deles no ano de 2018. Todos artigos são do tipo qualitativo evidenciando que o cuidado com paciente na terminalidade, necessita de uma investigação mais aprofundada, a qual é abarcada pela pesquisa qualitativa. O estudo qualitativo, objetiva além de conhecer as opiniões das pessoas sobre determinado tema, busca entender as motivações, os significados e os valores que sustentam as opiniões e as visões de mundo, ou seja, dar voz ao outro e compreender de que perspectiva ele fala (Fraser \& Godim, 2004).

Não foram encontrados artigos originados do estado do Rio Grande do Sul, tendo em vista que no estado no ano de 2018 houveram 6.210 casos de câncer de próstata, 5.110 casos de câncer de mama feminino, 840 casos de câncer de colo de útero, 2.760 casos de câncer de Traqueia, Brônquio e Pulmão em homens e 1.600 em mulheres (Brasil, 2018). Mostrando assim que mesmo com uma grande demanda de pacientes, pouco se pesquisa sobre a preparação dos profissionais para atuar na área, o que reflete diretamente no atendimento aos pacientes.

Dentre os cinco artigos, dois artigos tiveram como foco, analisar o cuidado paliativo dentro da terapia intensiva. $\mathrm{O}$ artigo publicado em 2017 que usou como amostra: enfermeiros, médicos, nutricionistas e fisioterapeutas, objetivou analisar o entendimento dos profissionais de saúde acerca da assistência ao paciente em cuidados ao fim da vida na unidade de terapia intensiva (UTI) oncológica, e discutir os objetivos que buscam alcançar ao planejar a assistência na perspectiva dos cuidados paliativos (Santos, Silva, Moreira, Zepeda, \& Gaspar, 2017). E o artigo publicado em 2018, também aplicado em UTI, com amostra de enfermeiros e técnicos de enfermagem tratou de conhecer o significado de cuidados paliativos ao idoso para a equipe de enfermagem e identificar como ocorrem as interações da família com o idoso na unidade de terapia intensiva. Foi o único dos cinco artigos analisados que teve como foco, o paciente idoso (Queiroz et al., 2018).

Em relação ao entendimento dos enfermeiros sobre Cuidado paliativo, o artigo de 2018 que contou com uma amostra de 13 enfermeiros buscou como objetivo além de evidenciar o entendimento dos enfermeiros sobre Cuidados Paliativos, também identificar os principais desafios encontrados pelos enfermeiros que cuidam de pacientes fora da possibilidade terapêtica e detectar o enfrentamento destes enfermeiros ao lidarem com essa clientela (Morais et al., 2018).

Quanto aos sentimentos que envolvem a assistência, o artigo de 2018 com amostra de 10 enfermeiros foi o único dos cinco analisados que tratou apenas dos sentimentos envolvidos na assistência, tendo como objetivo identificar os sentimentos de enfermeiros que atuam junto a pacientes com câncer em fase terminal. E este contou com uma psicóloga no grupo de pesquisadores (Alencar et al., 2017). Pensando que o tratamento envolve sentimentos além do paciente e da família, mas também do cuidador que presta a assistência.

Segundo Hercos et al. (2014), os enfermeiros atuantes na oncologia lidam diariamente com fatores estressantes por esta especialidade apresentar situações de contato com a morte, necessidade cuidados de alta complexidade e paliativos, bem como a atenção à família, constituindo grandes desafios para os profissionais. E sugere formas de lidar com as dificuldades minimizando-as ou resolvendo-as com além de melhoria das condições de trabalho, incentivo e inserção de atividades, físicas e de lazer no cotidiano dos profissionais, uma política de educação permanente, destaca-se o suporte psicológico sistematizado aos profissionais para o enfrentamento das dificuldades no seu cotidiano de trabalho e investimento na relação interpessoal na instituição.

No que tange os saberes dos acadêmicos de enfermagem com relação aos cuidados paliativos, o artigo publicado em 2017 com amostra de 16 acadêmicos de enfermagem, teve como objetivos identificar o saber dos acadêmicos de enfermagem com relação aos cuidados paliativos e discutir o diferencial deste profissional para a qualidade do cuidar ao cliente oncológico em estágio avançado, mostrando um interesse pelo conhecimento prévio antes do acadêmico graduar-se (Vieira et al., 2017). Isso vai ao encontro da importância das aulas práticas e estágios durante a graduação. 
Como resultado, em relação à importância dos cuidados paliativos, um dos artigos aplicados na UTI, o qual observou o foco no cuidado físico e não identificou destaque com a preocupação em ofertar cuidados paliativos pós alta da UTI, identificou através desta pesquisa a fragilidade no reconhecimento da importância dos cuidados paliativos correlacionando-os apenas aos pacientes em franca terminalidade de vida. Destacaram-se também dificuldades para prognosticar, identificar o paciente em cuidados paliativos, triar, decisão em equipe, planejamento do cuidado de forma integral com relação aos processos éticos e legais principalmente sobre a classe médica, carência na comunicação com familiares, déficit na formação do profissional relacionado aos cuidados paliativos e opiniões divergentes em relação às condutas terapêuticas (Santos et al., 2017).

Já outro estudo também aplicado no ambiente de UTI, constatou que os diferentes olhares em relação aos cuidados paliativos à pessoa idosa podem oportunizar um melhor cuidado ao paciente, mediante a um olhar compreensivo e interativo com os pacientes e familiares. Compreendeu-se que a equipe ao lidar com a terminalidade por meio dos cuidados paliativos, expressa imagens negativas sobre o tema com relação aos objetivos do ambiente de trabalho se fortalecem por ações de possibilidade de sobrevida, falta de experiência por se tratar de ações não condizentes entre terapia intensiva e cuidado paliativo (Queiroz et al., 2018). Pensando que o idoso tem maior suscetibilidade a doenças crônicas, incluindo o câncer. Afinal o envelhecimento natural do ser humano traz mudanças nas células, que as tornam mais vulneráveis ao processo cancerígeno. Isso, somado ao fato de as células das pessoas idosas terem sido expostas por mais tempo aos diferentes fatores de risco externos (presentes no ambiente) ou internos (hormônios, imunidade) para câncer, explica, em parte, o porquê de o câncer ser mais frequente nessa fase da vida (Brasil, 2018).

$\mathrm{O}$ artigo que teve como um dos objetivos evidenciar o entendimento dos enfermeiros sobre Cuidados Paliativos possibilitou verificar que a morte faz parte do cotidiano dos profissionais de enfermagem, porém com dificuldades em lidar e falar sobre a terminalidade da vida. Alguns profissionais responderam com negação, o que pode interferir na maneira de cuidado dos pacientes e familiares. Além do déficit de conhecimento de alguns profissionais, alguns mostraram também forte sensibilidade ao assunto, foi observado que os profissionais que apresentavam mais equilíbrio emocional diante dos desafios enfrentados foram os que possuíam maior experiência. Destacou que os cuidados paliativos originam muitos estudos que se referem à importância dos mesmos, mas poucos citam sobre a preparação dos profissionais atuais e futuros e como estão diretamente ligados ao processo. Acredita-se que a realização de estudos que exploram a experiência e o conhecimento da enfermagem profissionais em Cuidados Paliativos são de extrema relevância, bem como preencher esta lacuna científica promoverá assistência a profissionais relacionados a essa área (Morais et al., 2018).

$\mathrm{O}$ artigo publicado por cinco enfermeiros e uma psicóloga, concluiu que para o enfermeiro uma das maiores ansiedades enfrentadas é lidar com a morte, pois esta surge, na maioria dos casos, como um fenômeno doloroso e de difícil aceitação. A vivência do enfermeiro é, então, marcada por situações conflitantes, expondo a sentimentos, principalmente negativos, que podem causar danos que se refletem tanto nas relações emocionais quanto nos aspectos profissionais desse indivíduo, podendo induzir o enfermeiro ao afastamento da assistência direta ao paciente ou até mesmo ao abandono dos deveres profissionais nesta área. Portanto, acredita-se que deve haver apoio ao profissional da área oncológica por parte da instituição, como a formação de grupos de apoio ao profissional com a finalidade de compartilhar experiências e minimizar o sofrimento emocional. A instituição poderia ainda fornecer a oportunidade aos profissionais na participação de cursos de especialização e aperfeiçoamento de conhecimento, visto que há constante atualização tecnológica das modalidades terapêuticas (Alencar et al., 2017).

O conhecimento favorece o manuseio adequado do paciente sob condições críticas e reduz os sentimentos negativos do profissional em relação ao cuidado tornando-o consciente de que utilizou as habilidades possíveis e disponíveis no atendimento do paciente em questão. Este artigo contou com a participação de um profissional da psicologia no corpo de pesquisadores, o que permite uma abordagem ampla e coerente no que se refere aos sentimentos dos profissionais (Alencar et al., 2017). A qualidade de vida no trabalho está diretamente ligada ao suporte psicológico do trabalhador e estado emocional dos mesmos, 
principalmente dos profissionais da saúde. Por isso a participação de profissionais de psicologia em estudos que abordam a assistência do enfermeiro é de suma importância para uma abordagem completa.

$\mathrm{O}$ artigo publicado enfermeiros e acadêmicos de enfermagem obteve como categorias de resultados o conhecimento dos acadêmicos sobre o cuidado paliativo e a sua dificuldade em lidar com a morte; e o Papel do Enfermeiro no cuidado paliativo. E concluiu que a boa prática da enfermagem deve ser resultado de muita dedicação, de busca por conhecimentos científicos, e principalmente, pelo fortalecimento do ser humano que existe em cada profissional. E que esta característica é de extrema importância para a aplicação do sentimento no cuidar (Vieira et al., 2017).

Neste sentido, as aulas práticas/estágios têm a finalidade de levar o estudante a aperfeiçoar a técnica sobre conhecimentos de uma determinada disciplina teórica. Logo, consiste em inserir o discente em instituições de saúde para que, aprenda a realizar além de procedimentos, vivenciar casos reais de pacientes reais antes da graduação. Esse contato com os pacientes é imprescindível e deve permear a formação do profissional de enfermagem (Rodrigues, Rodrigues, Schönholzer, Rocha, \& Rocha, 2015).

\section{Considerações Finais}

Conclui-se a partir da análise dos artigos selecionados que apesar das pesquisas serem recentes (últimos dois anos) ainda se encontra lacuna no conhecimento referente a Preparação do enfermeiro frente a pacientes oncológicos em estado paliativo, uma vez que nenhum dos artigos publicados correlacionou diretamente a preparação técnica profissional e emocional dos profissionais. Partindo da premissa que todo profissional é um ser humano completo e é impossível separar suas relações profissionais e emocionais, estudar o binômio cuidados paliativos e enfermagem se faz cada vez mais pertinente. E que a preparação do enfermeiro segue intangível, uma vez que não se utiliza de métodos e estratégias para identificar o nível de preparação deste profissional.

Também se conclui que a preparação emocional e técnica para lidar com as situações da assistência do paciente oncológico em estado paliativo, provém do conhecimento, vivência e apoio psicológico que este deve ter para uma qualidade no atendimento e qualidade de vida do profissional. Desta maneira, se faz pertinente realizar pesquisas que abordem a preparação do enfermeiro frente a pacientes oncológicos em estado paliativo que abarque também a correlação entre técnica e suporte emocional, para que a assistência obtenha maior qualidade e que o profissional a realize com qualidade de vida.

\section{Referências}

Academia Nacional de Cuidados Paliativos. (2009). Manual de cuidados paliativos. Diagraphic. https://www.santacasasp.org.br/upSrv01/up_p ublicacoes/8011/10577_Manual\%20de\%20Cuidados\%20Paliativos.pdf

Alencar, D., Carvalho, A., Macedo, R., Amorim, A., Martins, Á., \& Gouveia M. (2017). Feelings of nurses who work with cancer patients in terminal phase. Revista de Pesquisa: Cuidado é Fundamental Online, 9(4), 1015-1020. http://www.seer.unirio.br/index.php/cuidadofundamental/article/view/5725. 10.9789/2175-5361.2017.v9i4.1015-1020

Bernardes, C., Bitencourt, J. V. O. V., Parker, A. G., Luz, K. R., \& Vargas, M. A. O. (2014). Percepção de enfermeira(o)s frente ao paciente oncológico em fase terminal. Revista Baiana de Enfermagem, 28(1), 31-41. 10.18471/rbe.v28i1.8883

Brasil. (2018). Ministério da Saúde. Instituto Nacional do Câncer. Cuidados Paliativos. INCA.

Floriani, C. A., \& Schramm, F. R. (2008). Cuidados paliativos: interfaces, conflitos e necessidades. Ciência \& Saúde Coletiva, 13(2), 2123-2132. 10.1590/S141381232008000900017

Fraser, M. T. D., \& Godim, S. M. G. (2004). Da fala do outro ao texto negociado: discussões sobre a entrevista na pesquisa qualitativa. Paidéia (Ribeirão Preto), 14(28), 139-152. 10.1590/S0103-863X2004000200004.

Hercos, T. M., Vieira, F. S., Oliveira, M. S., Buetto, L. S., Shimura, C. M. N., \& Sonobe, H. M. (2014). O trabalho dos profissionais de enfermagem em Unidades de Terapia Intensiva na assistência ao paciente oncológico. Revista Brasileira de Cancerologia, 60(1), 51-58. 
Research, Society and Development, v. 10, n. 3, e35210310642, 2020

(CC BY 4.0) | ISSN 2525-3409 | DOI: http://dx.doi.org/10.33448/rsd-v10i3.10642

Melo, A. G. C, \& Figueiredo, M. T. A. (2006). Cuidados paliativos: conceitos básicos, histórico e realizações da Associação Brasileira de Cuidados Paliativos e da Associação Internacional de Hospice e Cuidados Paliativos. In Pimenta, C. A. M., Mote, D. D. C. F., \& Cruz, D. A. L. M. (Coord.), Dor e cuidados paliativos: Enfermagem, Medicina e Psicologia. Barueri: Manole.

Monteiro, F. F., Oliveira, M., \& Vall, J. (2010). A importância dos cuidados paliativos na enfermagem. Revista Dor, 11(3), 242-248.

Morais, E. N., Conrad, D., Mattos, E. M., Cruz, S. A. C., Machado, G. C., \& Abreu, M. O. (2018). Cuidados paliativos: enfrentamento dos enfermeiros de um hospital privado na cidade do Rio de Janeiro - RJ. Revista de Pesquisa: Cuidado é Fundamental Online, 10(2), 318-325. http://www.seer.unirio.br/index.php/cuidadofundamental/article/view/6000. 10.9789/2175-5361.2018.v10i2.318-325

O Globo. (2015). Índice mostra que Brasil não cuida bem de pacientes terminais. https://oglobo.globo.com/sociedade/saude/indice-mostra-que-brasil-nao-cuidabem-de-pacientes-terminais-17697993

Queiroz, T. A., Ribeiro, A. C. M., Guedes, M. V. C., Coutinho, D. T. R., Galiza, F. T., \& Freitas, M. C. (2018). Cuidados paliativos ao idoso na terapia intensiva: olhar da equipe de enfermagem. Texto \& Contexto Enfermagem, 27(1). http://www.scielo.br/scielo.php?script=sci_arttext\&pid=S010407072018000100310\&lng=en. 10.1590/0104-07072018001420016.

Rodrigues, J. Z., Rodrigues, L. S., Schönholzer, T. E., Rocha, I. C., \& Rocha, E. M. (2015). A importância da aula prática na formação do profissional de enfermagem: um relato de experiência. Revista Panorâmica On-Line, 19, 99-110. http://oca.ufmt.br/revista/index.php/revistapanoramica/article/view/629/252

Santos, D. C. L., Silva, M. M., Moreira, M. C., Zepeda, K. G. M., \& Gaspar, R. B. (2017). Planejamento da assistência ao paciente em cuidados paliativos na terapia intensiva oncológica. Acta Paulista de Enfermagem, 30(3), 295-300. http://www.scielo.br/scielo.php?script=sci_arttext\&pid=S010321002017000300295\&lng=en. 10.1590/1982-0194201700045

Vieira, T. A., Oliveira, M., Martins E. R. C., Costa, C. M. A., Alves, R. N., \& Marta, C. B. (2017). Cuidado paliativo ao cliente oncológico: percepções do acadêmico de enfermagem. Revista de Pesquisa: Cuidado é Fundamental Online, 9(1), 175-180. http://www.seer.unirio.br/index.php/cuidadofundamental/article/view/5329. 10.9789/2175-5361.2017.v9i1.175-180 